\title{
Erziehung und demokratische Öffentlichkeit
}

\section{Ein vernachlässigtes Kapitel der politischen Philosophie}

\section{Axel Honneth}

Die Geschichte des öffentlichen Erziehungssystems in den demokratischen Verfassungsstaaten ist seit seinen Anfängen im 19. Jahrhundert eine ununterbrochene Kette von Konflikten um die Untergliederung, die Form und den Gehalt des schulischen Unterrichts gewesen; von den erbittert geführten Auseinandersetzungen, die entweder zwischen den Interessenvertretern sozialer Klassen oder einer organisierten Elternschaft und den staatlichen Behörden geführt wurden, blieben weder die Schichtung des Schulsystems noch die Lehrmethoden oder das Curriculum unberührt. Den Zündstoff, der in dem staatlichen Versprechen eines allgemeinen Bürgerrechts auf Bildung und Erziehung steckte, mag Immanuel Kant schon vorausgeahnt haben, als er in seinen Vorlesungen zur „Pädagogik“ den berühmten Satz formulierte: „Zwei Erfindungen der Menschen kann man wohl als die schweresten ansehen: die der Regierungs- und die der Erziehungskunst nämlich, und doch ist man selbst in ihrer Idee noch streitig. " Für Kant ergab sich die Parallele zwischen Regierungs- und Erziehungskunst aus der Überlegung, dass es sich bei beiden um gesellschaftlich geschaffene Einrichtungen handelt, die in den unterschiedlichen Dimensionen der Gattungs- und der Individualgeschichte, der Phylo- und der Ontogenese dieselbe Aufgabe zu leisten haben; sie müssen uns durch geschickte Wahl der Mittel und Methoden, eben durch eine Art von „Kunst“", darin unterrichten können, wie das eine Mal ein Volk von Untertanen, das andere Mal ein seiner Natur noch unterworfenes Kind aus dem Zustand der Unmündigkeit in den der Freiheit zu versetzen sei. Was aber zunächst nur nach einer bloßen Analogiebildung klingt, wird von Kant dann selbst im weiteren Verlauf seiner Vorlesungen noch viel stärker ausgedeutet, indem er auf ein Verhältnis der wechselseitigen Bedingung von republikanischer Staatsordnung und Erziehung verweist: Der kleine, naturgetriebene Mensch muss erst einen Prozess der auf Freiheit zielenden Erziehung durchlaufen haben, bevor er Mitglied eines sich selbst regierenden Staatsvolks werden kann, so wie umgekehrt nur autonome Bürgerinnen und Bürger eine öffentliche Erziehung institutionalisieren können, die ihren Kindern den Weg in die politische Mün-

\footnotetext{
Online publiziert: 07.07 .2012

(C) Die Autor(en) 2012. Dieser Artikel ist auf Springerlink.com mit Open Access verfügbar.
}

Festvortrag anlässlich der Eröffnung des 23. DGfE-Kongresses „Erziehungswissenschaftliche Grenzgänge" am 12. März 2012 in Osnabrück.

Prof. Dr. A. Honneth $(\bowtie)$

Institut für Sozialforschung, Johann Wolfgang Goethe-Universität, Senckenberganlage 26, 60325 Frankfurt am Main, Deutschland

E-Mail: honneth@em.uni-frankfurt.de 
digkeit ermöglicht. Eine gute Erziehung und eine republikanische Staatsordnung sind deswegen komplementär aufeinander angewiesen, weil jene in Form eines allgemeinen und öffentlichen Unterrichts im Einzelnen erst die kulturellen und moralischen Befähigungen hervorbringt, mit deren Hilfe diese dann derart existieren und gedeihen kann, dass die Bürgerschaft an der politischen Emanzipation auch des niederen Volkes noch Anteil nimmt. Wie schon im „Émile“ von Rousseau bildet daher in der „Pädagogik“ von Kant die Idee des ,guten Bürgers“" ${ }^{\text {"2 }}$ das Verbindungsglied zwischen Erziehungstheorie und Regierungslehre, zwischen Bildungskonzeption und politischer Philosophie: Ohne die eine wäre die andere nicht möglich, weil beide Voraussetzungen eines demokratischen Gemeinwesens erläutern, die unabhängig voneinander nicht existieren könnten.

Es ist diese äußerst enge, interne Verknüpfung gewesen, die im politisch-philosophischen Diskurs der Moderne dafür gesorgt hat, dass kaum ein Demokratietheoretiker von Rang und Namen nicht einen systematischen Beitrag zur Erziehungslehre verfasst hat; angefangen mit Rousseau und Kant über Schleiermacher bis hin zu Émile Durkheim und John Dewey haben sich immer wieder große und bedeutende Denker gefunden, denen es ganz selbstverständlich war, dem Thema der öffentlichen Erziehung eigenständige Monographien zu widmen. ${ }^{3}$ Die Pädagogik, verstanden als die Lehre von den Maßstäben und Methoden einer angemessenen Unterrichtung des Nachwuchses, wurde als Zwillingsschwester der Demokratietheorie begriffen, weil ohne ausgewogene Anleitungen dazu, wie im Kind Kooperationsfähigkeit und moralische Eigeninitiative zugleich zu wecken seien, gar nicht erläuterbar schien, was es heißen sollte, vom gemeinsamen Zusammenwirken in der demokratischen Selbstbestimmung zu sprechen; die Idee des ,guten Bürgers" war nicht Leerformel oder Zierde politischer Festreden, sondern praktische Herausforderung, der man sich durch den theoretischen Entwurf, ja die experimentelle Erprobung geeigneter Schulformen und Unterrichtsmethoden gewachsen zeigen musste. Als es daher nach der Zerschlagung des Nationalsozialismus darum ging, ein ganzes Volk wieder an die systematisch verlernten Praktiken der demokratischen Willensbildung zu gewöhnen, konnten etwa die amerikanischen Besatzungsmächte ganz selbstverständlich auf die pädagogischen Schriften ihres Landsmannes John Dewey zurückgreifen, um die vor ihnen stehende Aufgabe zu bewältigen; ${ }^{4}$ zwischen demokratischer Theorie und pädagogischer Praxis war das Band noch so eng, schien der interne Zusammenhang weiterhin so evident gegeben, dass es nichts von Paternalismus und Bevormundung an sich hatte, den demoralisierten Mitgliedern eines in Schutt und Asche versunkenen Unrechtsstaates demokratische Gepflogenheiten wieder beibringen zu wollen. Seither aber ist die Verknüpfung von Demokratie- und Erziehungskonzept, von politischer Philosophie und Pädagogik zerrissen; gewiss, es gibt immer wieder Vorstöße, über das Erfordernis einer demokratischen Erziehung nachzudenken, aber diese kommen meistens von einer alleingelassenen Erziehungswissenschaft und nicht mehr aus der Mitte der politischen Philosophie selbst. Die Demokratietheorie in der Vielzahl ihrer Gestaltungen und Stimmen schweigt sich über die erzieherische Seite ihres Geschäftes heute weitgehend aus, weder Überlegungen zu schulischen Methoden noch zum Lehrplan sind in ihr noch aufzufinden; jede Vorstellung davon, dass eine vitale Demokratie durch allgemeine Bildungsprozesse ihre eigenen kulturellen und moralischen Bestandsvoraussetzungen stets wieder erst erzeugen muss, ist der politischen Philosophie mittlerweile abhanden gekommen. Bevor ich im zweiten Schritt meines Beitrags darangehe, im Anschluss an die eben nur 
angedeutete Tradition den Zusammenhang von Erziehung und politischer Freiheit, von Bildung und Demokratie noch einmal zu erläutern (II), will ich in einem ersten Schritt zunächst versuchen, die Gründe für die inzwischen eingetretene Entkoppelung von Pädagogik und politischer Philosophie zu benennen; sie liegen, wie sich zeigen wird, in einem fatalen, „wahlverwandtschaftlich“ zu nennenden Zusammenschluss von problematischen Auffassungen über die kulturellen Voraussetzungen der Demokratie und einem falsch verstandenen Neutralitätsgebot des Staates (I). Nachdem ich auf diese Weise sowohl negativ als auch positiv für eine Wiederannäherung von Erziehungslehre und Demokratietheorie plädiert habe, will ich in einem dritten Schritt schließlich die Herausforderungen skizzieren, mit denen ein erneuertes Programm der demokratischen Erziehung heute vor allem konfrontiert ist; dabei muss ich es bei wenigen Stichworten bewenden lassen, weil jede ausführlichere Behandlung den zeitlichen Rahmen meines Beitrags sprengen würde (III).

Während Kant, Durkheim oder Dewey das Thema der demokratischen Erziehung noch als intrinsischen Bestandteil ihrer eigenen politisch-philosophischen Unternehmungen betrachteten, spielt es heute innerhalb der normativ orientierten Demokratietheorie nur noch eine randständige Rolle; zwar findet es hier gelegentlich noch einmal Erwähnung und wird in den entsprechenden Einführungen auch immer einmal wieder als Aufgabenfeld benannt, aber als solches wird dessen Erschließung, Bestimmung und Ausführung doch der wissenschaftlichen Pädagogik überantwortet. ${ }^{5}$ Die Demokratietheorie hat sich von ihrer Zwillingsschwester, der Lehre von der angemessenen Organisation und Methode einer demokratischen Bildung, verabschiedet und sich damit der Möglichkeit begeben, selbst etwas zur normativen Funktion von Vorschule, Schule und Erwachsenenbildung beisteuern zu können. Man könnte sich angesichts dieser Entwicklung mit der Feststellung beruhigen, dass sich darin nur das Ergebnis einer weiteren Differenzierung der einzelnen Wissenschaftsdisziplinen spiegelt, wie wir sie seit rund zweihundert Jahren beobachten können; so, wie sich die Philosophie in der zweiten Hälfte des 19. Jahrhunderts von der Gesellschaftslehre hat trennen müssen, die sich später dann im Fach der Soziologie hat etablieren können, musste sie sich im Laufe ihrer voranschreitenden Spezialisierung dann auch von der Aufgabe lösen, aus eigenen Mitteln die zur Herausbildung von demokratischer Mündigkeit erforderlichen Erziehungsprozesse zu bestimmen. Dass die Dinge nicht so einfach liegen und vielmehr auf ein schwerwiegenderes Problem verweisen, wird schon an dem Umstand ersichtlich, dass die politische Philosophie an jeder erdenklichen Stelle erneut auf das Problem der Erziehung stößt, ohne dafür heute auch nur den Ansatz einer Lösung bereitzuhalten. Mit der staatlichen Durchsetzung einer allgemeinen Schulpflicht ist der behördlich verwaltete Unterricht nämlich zu einem entscheidenden Hebel der Formung von Verhaltensweisen und Fähigkeiten der nachfolgenden Generation geworden, so dass er sowohl im Guten wie im Schlechten auf die Eigenschaften des zukünftigen Bürgers und der zukünftigen Bürgerin Einfluss nimmt. Die Art der schulischen Erziehung, ihre Methoden und Gehalte, kann sich entweder in wünschenswerter Weise auf den Bestand einer Demokratie auswirken, indem sie etwa Kooperationsvermögen und individuelle Selbstachtung fördert, oder aber in negativer 
Weise zu ihrer schleichenden Untergrabung beitragen, wo sie nämlich Autoritätshörigkeit und moralischen Konformismus vermittelt. ${ }^{6}$ Insofern bildete die Frage nach der angemessenen Form der staatlich organisierten Erziehung von Anfang an - man denke nur an Platons ,Politeia“7 - ein Herzstück jeder politischen Philosophie; wer sich damit beschäftigte, wie ein Staat oder ein politisches Gemeinwesen angesichts der menschlichen Natur beschaffen sein sollte, konnte und durfte nicht das damit zusammenhängende Problem aussparen, nach welcher Methode die schulische Erziehung welchen Schülern welche Gehalte beizubringen habe. Wenn diese Frage daher heute aus der politischen Philosophie weitgehend ausgeblendet und hier weder in ihrer positiven noch in ihrer negativen Gestalt weiterverfolgt wird, so kann das nicht einfach an der rational nachvollziehbaren Ausgliederung eines woanders viel besser und adäquater zu behandelnden Themas liegen; das Problem der staatlich organisierten Erziehung steht viel zu sehr im Zentrum allen politischen Handelns, berührt viel zu umfassend die Bestandsvoraussetzungen des demokratischen Rechtsstaats, als dass es sich heute so ohne Weiteres aus dem Korpus einer Wissenschaft oder Philosophie der Politik heraustrennen ließe.

Die Gründe, die helfen können, die wachsende Abkoppelung der Demokratietheorie von der Erziehungslehre zu erklären, müssen mithin auf einer anderen Ebene angesiedelt sein als die der voranschreitenden Differenzierung einzelner Fächer; ich vermute sie statt dessen auf einer weit tieferliegenden, begrifflichen Ebene, dort, wo es um die Bestimmung und Auslotung des Umfangs geht, in dem die Demokratie auf sich selbst einwirken kann. Als theoretische Faustregel mag hier gelten, dass dieser Umfang als um so geringer angesehen werden muss, je mehr an eigenen Bestandsvoraussetzungen dem demokratischen Rechtsstaat sei es aufgrund von normativen Beschränkungen oder aufgrund von sachlichen Unverfügbarkeiten entzogen zu sein scheinen; und wird schließlich, so ließe sich sagen, dieser Spielraum für selbstgenerative Aktivitäten für nur noch äußerst schmal gehalten, so wird folgerichtig auch der schulischen Erziehung kaum mehr ein politischer Stellenwert eingeräumt werden können, weil sich selbst mit ihrer Hilfe die Lebensbedingungen der Demokratie nicht verändern lassen. Es sind theoretische Wandlungen solcher Art, also Ernüchterungen über die selbstgenerativen Kräfte demokratischer Gemeinwesen, die meines Erachtens im Wesentlichen dafür verantwortlich sind, dass heute der Schule und der staatlich organisierten Erziehung eine nur noch geringe Aufmerksamkeit von seiten der politischen Philosophie zukommt. Ich will zwei der theoretischen Quellen benennen, die im Laufe der letzten Jahrzehnte dafür gesorgt haben mögen, warum die Demokratietheorie beinahe unmerklich und hinter ihrem Rücken den Glauben an den Wert der staatlich organisierten Erziehung für die Demokratie verloren hat; es ließen sich gewiss noch Einflussgrößen anderer Art benennen, etwa die Vorverlagerung der charakterlichen Prägung in die dem Staat entzogene Phase der frühkindlichen Sozialisation, aber ich will mich auf die beiden folgenden Aspekte beschränken, weil sie in das Hoheitsgebiet der politischen Philosophie selbst fallen.

Auf der einen Seite mag zur Verbreitung der Vorstellung, dass dem demokratischen Rechtsstaat ein nur sehr geringer Spielraum bei der Regenerierung seiner eigenen moralisch-kulturellen Bedingungen bleibt, die nach ihrem Autor als Böckenförde-These benannte Auffassung beigetragen haben, wonach die Demokratie in ihrer Reproduktion von der Zufuhr einiger ihr selbst vorausliegender Traditionsbestände abhängig ist. Obwohl der Staatsrechtler Böckenförde seine Einsicht ursprünglich wohl viel enger 
verstanden hat, nämlich als Hinweis auf die sittlichen Bestandsvoraussetzungen allein des modernen Rechts ${ }^{8}$ wird sie inzwischen doch viel weiter gedeutet und als Beleg für eine kulturelle Unselbständigkeit aller demokratischen Rechtsstaaten genommen: Solche politischen Systeme sind, folgt man dieser generalisierten Lesart, beständig darauf angewiesen, dass sie durch moralische Gewohnheiten und Verhaltensweisen kulturelle Unterstützung finden, für deren Wachstum und Pflege sie selbst nicht die geeigneten Instrumentarien besitzen. Auf der höchsten Stufe der Verallgemeinerung lautet die sogenannte Böckenförde-These heute dann sogar, dass Demokratien ihren sozialen Erhalt moralischen Einstellungen verdanken, die nur in gewachsenen Gemeinschaften mit ethisch-substanziellen, ja religiösen Orientierungen gedeihen können. Wird aber von einer derartigen Vorstellung ausgegangen, dann liegt die Schlussfolgerung nahe, den staatlich organisierten Erziehungsprozessen, also der schulischen und vorschulischen Bildung, allen Wert für die Vermittlung von demokratiefördernden Verhaltensweisen abzusprechen; denn was an moralischen Einstellungen der kooperativen Willensbildung entgegenkommt - Toleranzfähigkeit, das Sich-in-den-anderen-hineinversetzen-Können, Gemeinwohlorientierung -, wird nicht in wie gut auch immer durchgeführten Unterrichtsprozessen erlernt, sondern nur im ethischen Sozialisationsmilieu vorpolitischer Gemeinschaften. Wo sich bei Böckenförde zumindest noch Hinweise darauf finden, dass auch „Bildung und Erziehung ${ }^{69}$ dieses Werk verrichten können, bleibt in der allgemeinen Rezeption seiner These nur die Überzeugung vom Erfordernis demokratischer Gesellschaften übrig, auf das Überleben traditionaler Gesinnungsgemeinschaften zu vertrauen: Alle staatlichen Anstrengungen der Organisation einer demokratischen Erziehung sind vergeblich, weil sie nicht das an sittlichen Tugenden erzeugen können, was für den Fortbestand von Demokratien lebensnotwendig ist.

Mit dem Eindringen dieser Auffassung in den Theoriebestand der politischen Philosophie ist wohl eine der Voraussetzungen benannt, die in der jüngeren Demokratietheorie dazu geführt hat, Fragen nach der öffentlichen Erziehung weitgehend von sich fernzuhalten; derartige Problemstellungen müssen sich in dem Maße als überflüssig erweisen, in dem festzustehen scheint, dass demokratische Gesinnungen nicht in staatlich vermittelten Erziehungsprozessen, sondern in den vorpolitischen Milieus traditioneller Gemeinschaften erworben werden. Die populäre Version der Böckenförde-These stellt aber nicht den einzigen Grund dar, der heute die Demokratietheorie dazu bewegt, zur einstigen Zwillingsschwester, der Erziehungslehre, zunehmend auf Distanz zu gehen; solchen Tendenzen der Entkoppelung kommt vielmehr von einer anderen, stärker normativen Seite noch die Neigung entgegen, das staatliche Neutralitätsgebot derart restriktiv auszulegen, dass selbst noch die Prinzipien der demokratischen Willensbildung keinerlei Niederschlag im öffentlichen Schulunterricht mehr finden dürfen.

Wie schon die kulturkonservative Neudeutung der Demokratie, nach der diese nur unter permanenter Zufuhr von ihr vorausliegenden Traditionsbeständen lebensfähig bleibt, so hat sich auch die Verschärfung des Gebots staatlicher Neutralität eher im Rücken der politischen Philosophie vollzogen als zielgerichtet und bei vollem Bewusstsein; ja, es kann sogar sein, dass es sich bei dieser zweiten theoretischen Verschiebung um die unbeabsichtigte Konsequenz der insgesamt gutgemeinten Absicht handelt, den in unseren Gesellschaften wachsenden Pluralismus der ethnischen und religiösen Kulturen durch eine Unterstreichung der strikten Unparteilichkeit des staatlich organisierten Unterrichts 
Rechnung zu tragen. Natürlich unterliegt die politische Bestimmung des Tiefengrades staatlicher Neutralität seit jeher einem ständigen Fluss, in dem sich auf kaum durchsichtige Weise die ethischen Überzeugungen einer Mehrheit und damit auch sozialer Kräfteverhältnisse spiegeln; die Legitimität solcher Wertbindungen bemisst sich letztlich nur daran, ob sie dem Kriterium genügen, die universalistischen Grundsätze demokratischer Verfassungen nicht zu verletzen. Im immerwährenden Streit um die unvermeidbare Parteilichkeit staatlichen Handelns stand es für die von mir zuvor benannte Tradition von Kant bis Durkheim und Dewey stets außer Frage, dass der staatlich betriebene Unterricht genau die Werte zu verkörpern habe, die sich in der Entscheidung niedergeschlagen hatten, ihn für alle zukünftigen Staatsbürger verpflichtend zu machen: Das Recht der Eltern, ihren Kindern die je eigenen, partikularen Wertüberzeugungen zu vermitteln, musste an der Pforte der Schule gebrochen werden, damit den Zöglingen durch Einübung von reflexiven Verhaltensweisen der Weg zur Teilnahme an der öffentlichen Willensbildung geebnet werden konnte. Die Selbstverständlichkeit, die in einer solchen Ausrichtung des Schulunterrichts auf dieselben demokratischen Prozeduren zum Ausdruck gelangt, durch die er selbst erst als allgemein verpflichtend ins Leben gerufen worden war, wird aber heute zunehmend wieder in Zweifel gezogen; entweder besinnt man sich dabei auf das staatliche Neutralitätsgebot, um vor einer Überfrachtung der schulischen Erziehung mit ihr fremden, politischen Werten zu warnen, oder beklagt von interessierter Elternseite, dass bei zu starker Orientierung an demokratischen Zielen die Vermittlung von karrierefördernden Leistungen zu kurz kommen könne. Finden solche altgedienten Vorbehalte dann noch ungewollt öffentliche Unterstützung dadurch, dass angesichts des gewachsenen Multikulturalismus tatsächlich vieles für eine Befreiung unserer Schulen von weltanschaulichen Relikten spricht, so entsteht unmerklich jene diffuse Gemengelage von Falschem und Richtigem, aus der heraus plötzlich jede Art von Parteilichkeit des schulischen Unterrichts als gleichermaßen problematisch oder verwerflich gelten muss; das Gebot staatlicher Neutralität wird folgerichtig nun bis zu dem Punkt ausgedehnt, an dem selbst die Idee der demokratischen Erziehung ihre normative Selbstverständlichkeit verliert.

Gewiss ist die politische Philosophie der Gegenwart noch nicht im Ganzen von derart radikalisierten Interpretationen des Neutralitätsgebots staatlichen Handelns durchdrungen; in ihr wird vielmehr weiterhin heftig darüber gestritten, wie sich dieses Erbstück des liberalen Staatsdenkens angesichts der zunehmenden Heterogenität von kulturellen Wertvorstellungen angemessen reformulieren ließe. ${ }^{10}$ Aber im Verbund mit den kulturkonservativen Neudeutungen der Lebensfähigkeit von Demokratien zeichnet sich gegenwärtig doch eine theoretische Entwicklung ab, an deren Ende die Überzeugung stehen mag, dass die Methoden und Gehalte des staatlich verwalteten Unterrichts auch von allen demokratischen Zielsetzungen befreit werden müssten. Auf der Linie eines solchen denkbaren Fluchtpunkts liegen heute schon Erwägungen, die Schule nur noch mit der Aufgabe der Anerziehung eines ,zivilen Minimums“ zu beauftragen, ${ }^{11}$ Eltern durch Ausbildungsgutscheine die Wahl beim weltanschaulichen Charakter des Schulunterrichts zu überlassen ${ }^{12}$ und die Lehrerinnen und Lehrer insgesamt nicht mehr als Beauftragte des demokratischen Rechtsstaats, sondern nur noch als die der vereinigten Elternschaft zu verstehen. ${ }^{13}$ Je weiter diese zunächst nur tentativen Überlegungen aber vorangetrieben werden, je entschiedener also die öffentliche Schule als ethisch neutral gedacht würde, 
um an ihre Stelle eine Vielzahl von weltanschaulich gebundenen Privatschulen treten zu lassen, desto stärker ginge die demokratische Gesellschaft des beinahe einzigen Instruments verlustig, über welches sie zur Regenerierung ihrer eigenen moralischen Grundlagen verfügt; insofern ist der Konflikt um das staatliche Schulsystem, gleichgültig, ob er nun dessen Gliederung, die Curricula oder die verwendeten Methoden betrifft, immer auch ein Kampf um die Zukunftsfähigkeit von Demokratien. In keiner Tradition war das Wissen um diesen Zusammenhang deutlicher präsent als in derjenigen, die zunächst durch Kant angestoßen wurde und schließlich in Durkheim und Dewey ihren Höhepunkt fand.

\section{II}

Die bislang vorgetragenen Überlegungen haben in der bloß negativen Gestalt einer Kritik an Tendenzen der zeitgenössischen Demokratietheorie bereits indirekt zu erkennen gegeben, welche Prämissen vorausgesetzt werden müssen, wenn das öffentliche Schulsystem als eine notwendige Ergänzung, ja als die andere, ihr vorgelagerte Hälfte der demokratischen Willensbildung verstanden werden soll; weder darf dann, so haben wir gesehen, die praxisleitende Hypothese preisgegeben werden, dass auch jenseits der frühkindlichen Sozialisation und abseits von traditionsgestützten Sittlichkeiten die Fähigkeiten zur öffentlichen Deliberation erzieherisch geweckt werden können, noch darf leichtfertig das Recht des Verfassungsstaates aufs Spiel gesetzt werden, die von ihm organisierten Erziehungsprozesse mit demokratischen Bildungszielen zu versehen. Werden diese beiden negativen Prämissen ins Positive gewendet, so lässt sich auch sagen, dass unter den Aufgaben des demokratischen Rechtsstaates jene an vorderster Stelle zu stehen hat, jedem seiner zukünftigen Mitglieder durch angemessene Bildungseinrichtungen gleichermaßen die Möglichkeit an die Hand zu geben, an der öffentlichen Legitimierung seiner eigenen Entscheidungen „ohne Angst und Scham“ mitzuwirken. In Kants Erziehungsschrift, die stark unter dem Einfluss von Rousseaus „Émile“ steht, ohne ihm freilich in allem zu folgen, nimmt die damit umrissene Idee vorläufig nur die Gestalt an, gegen die „häusliche“ die allgemein zugängliche, „öffentliche“ Erziehung zu verteidigen, deren Vorteil es sei, ohne die Gefahr der Fortsetzung von „Familienfehlern“ in jedem Zögling die Tugenden und Fähigkeiten des „,künftigen Bürgers“ hervorzubringen; ${ }^{14}$ alles, was in solchen Erziehungsprozessen pädagogisch vermittelt werden soll - erstens mechanische Geschicklichkeiten, zweitens pragmatische Klugheiten und drittens moralische Selbstbestimmung -, untersteht daher für Kant interessanterweise dem primären Ziel, im Einzelnen ebenso viele Schichten der Selbstachtung und des Selbstwerts entstehen zu lassen, die ihm zusammengenommen dann ein selbstbewusstes Auftreten als Bürger einer Republik erlauben. ${ }^{15}$ Die Frage danach, welchen Beitrag die drei Klassen von anerziehbaren Fähigkeiten dann später zur Berufsausübung leisten sollen, stellt sich in diesem Zusammenhang noch gar nicht, weil sie alle gleichermaßen unter dem alleinigen Gesichtspunkt des Erwerbs von verschiedenen Formen des Selbstwertgefühls betrachtet werden; der zukünftige Staatsbürger muss, so heißt es bei Kant wie in einer Vorwegnahme eines berühmten Gedankens von John Rawls, ${ }^{16}$ zunächst über das zentrale Gut der „Selbstachtung“ verfügen können, bevor er sich als Gleicher unter Gleichen an der republikanischen Selbstgesetzgebung beteiligen kann. Berufliche Geschicklichkeiten, zivilgesellschaftliches Orientierungswis- 
sen und moralische Prinzipien werden daher gerade nicht primär als erlernbare Mittel zu späterer Einkommenssicherung, sondern als gesellschaftlich verallgemeinerte Medien der sozialen Anerkennung begriffen, über deren pädagogisch vermittelte Aneignung der Heranwachsende schrittweise zu dem Bewusstsein gelangen soll, in den Augen seiner Mitmenschen einen „Wert“ zu besitzen: Das Erlangen von Geschicklichkeiten gewährt ihm, wie es Kant nahezu in den Worten Hegels formuliert, Respekt ,in Ansehung seiner selbst als Individuum“, der Erwerb von zivilgesellschaftlichem Wissen den ,öffentlichen Wert" eines Bürgers, und die Aneignung der moralischen Prinzipien schließlich die Achtung ,in Ansehung des ganzen menschlichen Geschlechts“"17.

Bei aller Hellsichtigkeit in Bezug auf den Zusammenhang von allgemeiner Schulbildung und reziproker Anerkennung im republikanischen Gemeinwesen ist Kant allerdings noch weit davon entfernt, daraus auch schon Rückschlüsse zu ziehen in Hinblick auf die Methode und die Organisationsform des schulischen Unterrichts; ihm steht der einzelne, männliche Zögling vor Augen, der je für sich durch erzieherisch aufgenötigten Wissenserwerb zur Selbstachtung gelangen soll, nicht aber die kooperative Gemeinschaft, in der jeder als anerkanntes Mitglied mit allen anderen zum Zweck der gemeinsamen Willensbildung zusammenwirken können muss. Zwar ahnt er in seiner „Pädagogik“ gelegentlich bereits, dass die Republik viel stärker noch als auf eine einseitige Wissensvermittlung auf die Schulung der kommunikativen Tugenden angewiesen wäre, ${ }^{18}$ gleichwohl schreckt er noch vor der Konsequenz zurück, in der öffentlichen Schule zunächst und vor allem eine Bildungsstätte demokratischer Fähigkeiten auszumachen. Diesen Schritt vollziehen erst rund einhundert Jahre später Émile Durkheim und John Dewey, als sie jeweils in ihrem eigenen Werk darangingen, den inneren Zusammenhang von Erziehung und Demokratie, von schulischen Lernprozessen und zukünftiger Staatsbürgerrolle zu umreißen.

Obwohl die Grundannahmen der beiden Denker einander nahezu auszuschließen scheinen - hier der szientistisch gesinnte Soziologe, dort der pragmatisch verfahrende Philosoph -, weisen ihre Überlegungen zur demokratischen Erziehung doch eine Reihe von überraschenden Gemeinsamkeiten auf. Unter den drei Funktionen, die die Schule aus heutiger Sicht in sich zu bündeln hat, die der Berufsqualifikation, die des Ausgleichs familien- oder milieubedingter Bildungsdefizite und die der allgemeinen Vorbereitung auf die Staatsbürgerrolle, heben Durkheim und Dewey einzig und allein auf die dritte, die zuletzt genannte ab; wie schon Kant verstehen sie das Erlernen von beruflich verwertbarem Wissen eher als ein beiläufiges Resultat der Einübung von demokratischen Gewohnheiten; und alles, was an kompensatorischer Erziehung zu leisten ist, wird als selbstverständliche Aufgabe der jeweiligen Schulgemeinschaft begriffen. Auch das Recht des demokratischen Staates, das von ihm durch Steuereinnahmen finanzierte Erziehungssystem an die Auflage zu binden, staatsbürgerliche Fähigkeiten zu regenerieren, wird von beiden Denkern, wo sie es überhaupt für nötig halten, mit ähnlichen Argumenten begründet. Weil sich die staatliche Einführung der allgemeinen Schulpflicht nur unter der Bedingung als ein demokratisch legitimationsfähiger Akt deuten lässt, dass sie als eine gemeinsame, gleichsam zivilgesellschaftliche Anstrengung gewertet wird, allen Bürgerinnen und Bürgern gleichermaßen zur faktischen Ausübung ihrer politischen Rechte zu verhelfen, darf diesem Staat nun nicht nachträglich das Recht abgesprochen werden, den schulischen Unterricht auch tatsächlich zur Einübung der entsprechenden Kenntnisse und Praktiken zu nutzen. ${ }^{19}$ 
Aber solche demokratietheoretischen Überlegungen bilden nur den allgemeinen Rahmen, in dem Durkheim und Dewey nun die Untersuchungen ansiedeln, die sie dem Gehalt und der Struktur des schulischen Unterrichts widmen. Den Ausgangspunkt ihrer wiederum stark übereinstimmenden Argumentationen stellt die These dar, dass die Vorbereitung auf die zukünftige Staatsbürgerrolle weniger eine Sache der angemessenen Wissensvermittlung ist als vielmehr der praktischen Gewohnheitsbildung; was die Schülerinnen und Schüler im schulischen Unterricht erlernen sollen, um später effektiv an der demokratischen Willensbildung teilnehmen zu können, sind nicht primär überprüfbare Kenntnisse über politische oder geschichtliche Zusammenhänge, sondern Verhaltensweisen, welche das moralisch selbstbewusste Auftreten in einer kooperierenden Gemeinschaft erlauben. Dass John Dewey als Pragmatist seine Überlegungen in eine derartige Richtung lenkt und also die Schule zuvorderst als eine Bildungsstätte des Zusammenwirkens in der forschenden Öffentlichkeit begreift, mag nicht sonderlich überraschen; ${ }^{20}$ aber dass Émile Durkheim ähnlich argumentiert und die Schule ebenfalls unter dem Blickwinkel betrachtet, welchen Beitrag sie zur Einübung demokratischer Kooperationsfähigkeiten leistet, bedarf wohl einer kurzen Erläuterung.

Durkheim lässt sich zwar zunächst im Unterschied zu Dewey, der hier weitaus optimistischer dachte, von der an Kant orientierten Vorstellung leiten, dass in jedem Kind die egoistischen Neigungen erst durch moralische Disziplinierungen gebrochen werden müssen, bevor es sich autonom an die sozialen Regeln der demokratischen Gemeinschaft zu halten lernt; aber er weicht doch darin erheblich von seinem philosophischen Lehrmeister $\mathrm{ab}$, dass er diesen vorausliegenden Erziehungsprozess um so geschmeidiger und vor allem um so erfolgversprechender vonstatten gehen sieht, je stärker dabei durch praxisnahe Vorbilder und spielerische Aktivitäten auch die Leidenschaften und Begehrlichkeiten, kurz, die sinnliche Natur des Kindes, angesprochen werden. ${ }^{21}$ Im Grunde genommen stellt daher für Durkheim das Aufbrechen des kindlichen Egoismus gerade nicht, obwohl er immer wieder davon spricht, einen Prozess der moralischen Disziplinierung dar, sondern einen Vorgang der affektvermittelten Gewohnheitsbildung: Das Kind lernt durch Teilnahme an ihm gemäßen, seine Neigungen also affizierenden Praktiken zunächst einmal nur, jene Regeln des demokratischen Zusammenlebens habituell zu beherrschen, die es sich dann später mit wachsendem Alter auch in ihrem rationalen Geltungsanspruch klarzumachen hat. Für die Schule und ihre Erziehungsstile kann Durkheim dann aber aus diesen Korrekturen Kants beinahe dieselben Konsequenzen ziehen, zu denen Dewey im Ausgang von seinen ganz anderen, nämlich hegelianischen Prämissen, gelangt war: Im schulischen Unterricht müssen die Heranwachsenden durch möglichst kooperative Lernmethoden, durch Mitbestimmung an allen die Schule betreffenden Angelegenheiten und schließlich durch eher gemeinschaftsbezogene als individuelle Formen des Tadels und der Ermutigung daran gewöhnt werden, sich schon früh jenen Geist des demokratischen Zusammenwirkens anzueignen, der ihnen im Erwachsenenalter dann zu einem selbstbewussten Auftreten in der politischen Öffentlichkeit verhelfen kann. Insofern sind beide Denker auch nicht der heute häufig vertretenen Meinung, dass die Schule primär der Ausbildung von individueller Autonomie zu dienen hat; ihr Erziehungsbild ist vielmehr übereinstimmend von der Idee geprägt, den Schülern ein sicheres Gespür dafür beizubringen, was es heißt, den Mitschüler als einen gleichberechtigten Partner in einem gemeinsamen Lern- und Untersuchungsprozess zu verstehen. Soll die öffentliche Schule 
in jeder nachwachsenden Generation erneut diejenigen Verhaltensweisen erzeugen, die für die demokratische Willensbildung lebensnotwendig sind, so muss sie statt auf die einseitige Vermittlung von moralischen Prinzipien viel stärker auf die Eingewöhnung in eine Kultur der Assoziation setzen; ${ }^{22}$ nicht das Erlernen von individuellen Grundsätzen richtigen Handelns, sondern das kommunikative Einüben von Perspektivübernahme und moralischer Initiative bilden für Durkheim und Dewey den Königsweg, auf dem der schulische Unterricht zur Regenerierung der Demokratie beitragen kann.

Nun ist allerdings nicht nur zwischen Kants Pädagogik und diesen Formulierungen bereits ein Jahrhundert vergangen, sondern zwischen ihnen und unserer Gegenwart noch einmal dieselbe Anzahl an Jahren. Angesichts des großen Zeitabstands mag daher so mancher den Kopf schütteln und mit triumphalistischer Geste darauf verweisen, dass sich inzwischen doch mit der sozialen und ökonomischen Umwelt auch die Anforderungen an die Schule radikal verändert haben: Die enorm gewachsenen Schülerzahlen, der wirtschaftliche Ruf nach Flexibilität und Leistungsbereitschaft, die trotz aller sozialpolitischen Anstrengungen unverändert fortwirkenden Bildungsdefizite der unteren Schichten, all das scheint in den kapitalistisch hochentwickelten Ländern keine andere Wahl zu lassen, als im Schulunterricht verstärkt auf Selektionsdruck, individuelle Leistungskontrolle und Anreiz zum Konkurrenzverhalten zu setzen. ${ }^{23}$ Schon gehen in den USA Politiker, Wirtschaftsfachleute und Manager daran, eine Reform des gesamten Schulsystems vorzuschlagen, die in der Summe der angekündigten Maßnahmen auf nichts anderes hinausliefe als eine Aktivierung des Unterrichts zugunsten des Erwerbs von rein ökonomisch verwertbaren Fähigkeiten: Die Effektivität aller amerikanischen Schulen soll nach diesen Plänen vergleichend anhand von quantitativen Daten kontrolliert werden, die den Erfolg der einzelnen Lehrer daran messen, zu welchem Abschneiden sie ihren Schülern bei den landesweit standardisierten Tests zu verhelfen vermochten. ${ }^{24}$ Kontrollverfahren solcher Art, die über kurz oder lang die Marginalisierung aller nicht statistisch überprüfbaren Fächer zur Folge hätten, werden mittlerweile aber nicht nur in den USA, sondern auch auf dem Kontinent diskutiert, so als gälte es auch hier, die Schulen mit Hilfe finanzwirtschaftlicher Methoden einseitig dem Diktat der ökonomischen Entwicklung zu unterwerfen. Für die Erinnerung an Zeiten, in denen der öffentliche Schulunterricht einmal als Dreh- und Angelpunkt der Selbsterneuerung von Demokratien betrachtet wurde, scheint unter derartigen Umständen kein Bedarf zu bestehen; nicht nur die Demokratietheorie selbst, sondern auch die staatliche Politik hat offenbar das Interesse an dem einzigen Organ verloren, mit dem sich wenigstens versuchsweise und bei steter Anstrengung die fragilen Voraussetzungen einer demokratischen Willensbildung des Volkes immer wieder regenerieren ließen.

Diesen Tendenzen zu einer Abkehr von der Idee der demokratischen Erziehung widersprechen freilich alle empirischen Befunde, die die vergleichende Bildungsforschung und die verschiedenen Pisa-Studien in den letzten Jahren zu Tage gefördert haben; denn darin findet auf eine geradezu wundersame Weise Bestätigung, was Durkheim und Dewey vor einem Jahrhundert vorausgedacht hatten, als sie zwischen kooperativen, demokratiefördernden Lehrmethoden und schulischen Leistungen eine ganz enge Beziehung herstellen wollten. Das Schulsystem, das bei allen internationalen Leistungsvergleichen stets die besten Ergebnisse erzielt, ist nämlich zugleich dasjenige, in welchem die demokratischen Ideale der beiden Denker noch am ehesten zur Verwirklichung gelangt sind: In 
den finnischen Schulen bleiben die Schüler unterschiedlicher Bildungsherkünfte so lange wie nur möglich in einer einzigen Schulgemeinschaft zusammen, werden Test- und Prüfungsverfahren auf das nur eben erforderliche Minimum reduziert, wird kommunikativer Verantwortung und wechselseitigem Vertrauen weit mehr Gewicht beigemessen als individueller Zurechenbarkeit und gehört schließlich die Hoheit über die Gestaltung des Unterrichts allein einer professionell ausgebildeten, mit den Schülervertretern eng kooperierenden Lehrerschaft. ${ }^{25}$ Gewiss, in den Beschreibungen des Schulsystems in Finnland wird nicht das Vokabularium Durkheims und Deweys verwendet, da ist weder die Rede von Gewohnheitsbildung noch von Gemeinschaftsmoral oder kooperativen Erziehungsmethoden; aber solche Rückübersetzungen in eine Sprache der demokratischen Bildung scheinen doch leicht möglich und gäben dann den seltenen, historisch unwahrscheinlichen Glücksfall zu erkennen, dass das politisch und normativ Richtige mit dem pragmatisch Zweckmäßigen einmal zusammenfiele: Jene Schulformen sind für die kognitiven Leistungen und Fähigkeiten der Schüler die besten, die zugleich auch der Regenerierung demokratischer Verhaltensweisen am stärksten entgegenkommen. Es gibt daher in Zeiten, in denen allerorten von wachsender politischer Apathie gesprochen und sogar die Gefahr einer „Postdemokratie“ an die Wand gemalt wird, ${ }^{26}$ keinen, aber auch keinen Grund, nicht die von Kant, Durkheim und Dewey begründete Tradition noch einmal wiederzubeleben und die öffentliche Erziehung als zentrales Organ der Selbstreproduktion von Demokratien zu begreifen.

\section{III}

Nach dem bislang Gesagten könnte leicht der Eindruck entstehen, als seien zugleich mit den angemessenen Methoden eines demokratischen Unterrichts auch dessen Stoffe ein für allemal gegeben und damit jedem historischen Wandel auf Dauer entzogen; vor allem Kant hat dieser Vorstellung starken Auftrieb gegeben, bindet er doch den Erwerb der für den Bürger erforderlichen Formen der Selbstachtung an das stufenweise Erlernen zeitlos gültiger Wissensgehalte; aber auch bei Durkheim lässt sich die Neigung ausmachen, bestimmte Materien allen Prozessen der kooperativen Erziehung als fixe Bestandteile vorzuschreiben. ${ }^{27}$ Nur John Dewey widerspricht in seinen pädagogischen Schriften der damit umrissenen Tendenz, indem er immer wieder mit großer Nüchternheit hervorhebt, dass sich mit den jeweiligen Herausforderungen der öffentlichen Problemlösung auch die stofflichen Gehalte des Unterrichts wandeln müssen; zwar geht auch er wie sein Mitstreiter Durkheim von einer unverzichtbaren Hierarchisierung der zu vermittelnden Wissensdisziplinen aus - in seinem Fall nehmen erstaunlicherweise die Geographie und die Geschichte komplementär den obersten Rang ein $-{ }^{28}$ was jedoch in diesen Disziplinen durch kooperatives Zusammenwirken jeweils erlernt werden muss, bemisst sich für ihn einzig und allein an den sich historisch verändernden Aufgaben der demokratischen Willensbildung. ${ }^{29}$ Ich will meinen Beitrag nicht beenden, ohne im Geiste Deweys nicht wenigstens die zwei geschichtlichen Herausforderungen stichwortartig benannt zu haben, die sich heute aufgrund ihrer Folgewirkungen für das demokratische Zusammenleben vor allem im Unterrichtsstoff niederzuschlagen hätten; von beiden Problematiken dürfte sich bei ausführlicherer Betrachtung zeigen, dass sie nicht durch Abschiebung in ein einzelnes 
Fach angemessen behandelt werden können, sondern eine stoffliche Mitthematisierung in fast dem gesamten Fächerkanon unserer Schulen erforderlich machen.

Für jeden aufmerksamen Zeitgenossen dürfte gegenwärtig außer Frage stehen, dass die digitale Revolution der Kommunikationsverhältnisse nicht nur die Formen der Anbahnung und Aufrechterhaltung privater Beziehungen, sondern auch die Wege der politischen Meinungsbildung nachhaltig verändern wird; mit dem Internet, das den Einzelnen in die Lage zugleich einer Enträumlichung und Beschleunigung seiner Interaktionen versetzt, entstehen heute mit wachsendem Tempo eine Vielzahl von Netzöffentlichkeiten, deren Außengrenzen und Themen im ständigen Fluss begriffen sind ${ }^{30}$ Es ist gewiss die Aufgabe des schulischen Unterrichts, die Schüler auf den Gebrauch dieses neuen Mediums technisch und sozial vorzubereiten, aber darin dürfte sich die gemeinsame Bewältigung seiner historischen Folgen bei Weitem nicht erschöpfen; vielmehr schiene es mir darüber hinaus vonnöten, in der experimentellen Überprüfung des Zustandekommens digital verbreiteter Themen und Wissensbestände gemeinsam zu erkunden, wo neben den Potenzialen auch die Grenzen und Gefährdungen des neuen Mediums liegen. Bei solchen exemplarischen Rekonstruktionen, in denen etwa die Genese und die Qualität bestimmter Lexikoneinträge bei Wikipedia unter die Lupe genommen werden könnten, müssten die verschiedenen Schulfächer eng zusammenarbeiten, weil neben dem rein technischen und ökonomischen Sachverstand auch Kenntnisse in den entsprechenden Themengebieten gefragt wären. Aber dieses eine Beispiel soll hier nur für die viel allgemeinere Idee stehen, dass es heute in unseren Schulen darauf ankäme, zugleich mit der Handhabung des Internets auch dessen Produktionsgesetze und Wirkungsweisen zu erlernen; die Schülerinnen und Schüler müssten durch die kooperative Nutzung des Computers, also ganz im Sinne Deweys, darauf vorbereitet werden, sich später einmal mündig der neuen Instrumente der politischen Willensbildung zu bedienen.

Stellt die digitale Revolution die erste der beiden geschichtlichen Herausforderungen dar, vor welche die demokratische Öffentlichkeit heute gestellt ist, so bildet die wachsende Heterogenität der Bevölkerung in den westlichen Ländern deren zweite. Wie in Bezug auf das neue Medium des Internets, so dürfte auch mit Blick auf den Multikulturalismus inzwischen kaum ein Dissens darüber bestehen, dass in den Schulen alles unternommen werden muss, um auf diese veränderten Bedingungen der öffentlichen Meinungsbildung vorzubereiten; aber auch hier stellt sich wieder die Frage, wie die methodischen Mittel und materialen Gehalte beschaffen sein sollten, die die geforderte Aufgabe am besten zu lösen vermögen. Die Idee der demokratischen Erziehung, an die ich mit meinem Beitrag erinnern wollte, hält für den ersten, methodischen Teil dieser Frage bereits die Antwort bereit: Je weniger die Schüler oder Schülerinnen im Unterricht als isolierte, leistungserbringende Subjekte adressiert, je stärker sie mithin als Mitglieder einer lernenden Kooperationsgemeinschaft behandelt werden, desto eher dürften sich unter ihnen Kommunikationsformen einstellen, in denen kulturelle Differenzen nicht nur spielerisch akzeptiert, sondern als Chancen der wechselseitigen Bereicherung begriffen werden können. Allerdings ist mit diesem Verweis auf die anerkennungsstiftende Kraft der demokratischen Erziehung noch nicht die zweite, materiale Teilfrage beantwortet, in der es darum geht, welche Konsequenzen aus dem wachsenden Multikulturalismus unserer Gesellschaften für den Unterrichtsstoff selbst gezogen werden müssen. Lassen Sie mich mit der Vermutung schließen, dass wir uns alle noch gar nicht angemessen ausmalen 
können, wie bei einer fairen Berücksichtigung der ethnischen und kulturellen Zusammensetzung der Schulklassen jene zu erlernenden Gehalte in fünfzehn oder zwanzig Jahren beschaffen sein müssen; sollen diese künftigen Schülerinnen und Schüler einmal zu mündigen Teilnehmern einer dann äußerst heterogenen, bunt zusammengewürfelten Öffentlichkeit werden, so müssen sie sich die Geschichte, die Literatur, die Geographie und die meisten anderen Fächer aus derselben dezentrierten Perspektive anzueignen lernen, die wir uns heute an den Universitäten in den entsprechenden Disziplinen erst allmählich und mühsam beizubringen versuchen.

\section{Anmerkungen}

1 Kant 1964, S.703.

2 Kant 1964, S.760.

3 Schleiermacher 2000; Durkheim 1973; Dewey 1993.

4 Zur „Reeducation“vgl. Gagel 2005, Kap. 2.

5 Eine große Ausnahme bildet die politische Philosophin Amy Gutmann, für die allerdings die hier benannte Tradition eine untergeordnete Rolle spielt: vgl. Gutmann 1999.

6 In dieser ,negativen“ Rolle taucht die Schule etwa in der politischen Philosophie von Louis Althusser auf: vgl. Althusser 1973, besonders S. $140 \mathrm{ff}$.

7 Platon 1990; Bd.IV, v. a. Buch V. Vgl. dazu auch Dewey 1993, S. 122-126.

8 Vgl. etwa Böckenförde 2006, S. 42-57.

9 Böckenförde 2006, S. 48.

10 Rawls 1992, S. 364-397; hilfreich ist auch Forst 1994, S. 78-83.

11 Vgl. dazu Gutmann 1999, S. 292-303.

12 Gutmann 1999, S. 65 f.; Walzer 1992, S. 314-318.

13 Zu solchen Tendenzen vgl. sehr gut: Dubet 2002, S. 101-104. Die Idee, dass Lehrerinnen und Lehrer sich als Beauftragte des demokratischen Rechtsstaates verstehen müssen und gerade nicht als Agenten der Eltern in einer fortgeschrittenen Lebensphase der Kinder, stammt von Durkheim (1973, S. 187-198). Zu dieser Problematik vgl. auch Gutmann 1999, S. 292-303.

14 Kant 1964, S.710-712. Beinahe gleichlautend heißt es bei Schleiermacher (2000, Bd.1, S. 272f.): „Wir finden Zeiten in der Geschichte unserer neuen Welt, wo Völker nur dadurch aus einer langen Dumpfheit und Rohheit zu erwachen scheinen, daß ihre Regierung die Zügel dieses wichtigen Geschäftes in die Hand nimmt und durch andere Mittel in dem jüngeren Geschlecht die gewünschten höheren Kräfte aufzuregen sucht, welche das ältere auf dem gewöhnlichen Wege der häuslichen Erziehung deshalb nicht zu erwecken vermag, weil sie in ihm selbst nicht vorhanden oder erstorben sind."

15 Kant 1964, S.713.

16 Vgl. Rawls 1979, S. 479-486.

17 Kant 1964, S. 713.

18 Bei Kant heißen diese demokratischen Fähigkeiten „weltbürgerliche Gesinnungen“ und tauchen nur gelegentlich in seiner „Pädagogik“ auf; vgl. etwa Kant 1964, S. 761.

19 Zu solchen Argumenten vgl. auch Gutmann 1999.

20 Vgl. zur Pädagogik Deweys insgesamt: Oelkers 2009. 
21 Durkheim 1973, S. $158 \mathrm{f}$.

22 Zur Verteidigung dieser Idee einer „Assoziationsmoral“ als richtungsweisend für die Schule vgl. auch Gutmann 1999, S. 59-64.

23 Vgl. exemplarisch Dubet 2002, Kap. 3; vgl. auch Dubet 2000.

24 Ravitch 2012.

25 Sahlberg 2012.

26 Crouch 2008.

27 Durkheim 1973, 17. und 18. Vorlesung.

28 Dewey 1993, 16. Kap. (S. 274-288).

29 Dewey 1993, S. $254 \mathrm{ff}$.

30 Honneth 2011, S. 560-567.

Open Access: Dieser Artikel unterliegt den Bedingungen der Creative Commons Attribution License. Dadurch sind die Nutzung, Verteilung und Reproduktion erlaubt, sofern der/die Originalautor/en und die Quelle angegeben sind.

\section{Literatur}

Althusser, L. (1973). Ideologie und ideologische Staatsapparate. In L. Althusser (Hrsg.), Marxismus und Ideologie (S. 111-172). Westberlin: VSA.

Böckenförde, E.-W. (2006). Freiheit und Recht, Freiheit und Staat. In E.-W. Bockenförde (Hrsg.), Recht, Staat, Freiheit (4. Aufl., S. 42-57). Frankfurt a. M.: Suhrkamp.

Crouch, C. (2008). Postdemokratie. Frankfurt a. M.: Suhrkamp.

Dewey, J. (1993). Demokratie und Erziehung. Eine Einleitung in die philosophische Pädagogik. Weinheim: Beltz.

Dubet, F. (2000). L'égalité et le mérite dans l'école démocratique de masse. L'Année sociologique, $50(2), 383-408$.

Dubet, F. (2002). Le Déclin de l'Institution. Paris: Seuil.

Durkheim, E. (1973). Erziehung, Moral und Gesellschaft. Neuwied: Luchterhand.

Forst, R. (1994). Kontexte der Gerechtigkeit. Frankfurt a. M.: Suhrkamp.

Gagel, W. (2005) Geschichte der politischen Bildung in der Bundesrepublik Deutschland 19451989/90 (3. Aufl.) Wiesbaden: VS Verlag für Sozialwissenschaften.

Gutmann, A. (1999) Democratic education (revised ed.). Princeton: Princeton Univ. Press.

Honneth, A. (2011). Das Recht der Freiheit. Grundriß einer demokratischen Sittlichkeit. Frankfurt a. M.: Suhrkamp.

Kant, I. (1964). Über Pädagogik. In I. Kant (Hrsg.), Werke in zwölf Bänden (Bd. XII). Frankfurt a. M.: Suhrkamp.

Oelkers, J. (2009). John Dewey und die Pädagogik. Weinheim: Beltz.

Platon (1990). Politeia. In Platon (Hrsg.), Werke in acht Bänden (Hrsg. v. G. Eigler, 2.Aufl., Bd. IV). Darmstadt: Wissenschaftliche Buchgesellschaft.

Ravitch, D. (2012). Schools we can envy. New York Review of Books, LIX(4), $19 \mathrm{f}$.

Rawls, J. (1979). Eine Theorie der Gerechtigkeit. Frankfurt a. M.: Suhrkamp.

Rawls, J. (1992). Der Vorrang des Rechten und die Ideen des Guten. In J. Rawls (Hrsg.), Die Idee des politischen Liberalismus. Aufsätze 1978-1989 (S.364-397). Frankfurt a. M.: Suhrkamp.

Sahlberg, P. (2012). Finnish lessons: What can the world learn from educational change in Finland? New York: Teachers College, Columbia Univ.

Schleiermacher, F. (2000). Über den Beruf des Staates zur Erziehung. In F. Schleiermacher (Hrsg.), Texte zur Pädagogik. Kommentierte Studienausgabe. (Hrsg. v. M. Winkler \& J. Brachmann, Bd. 1, S. 272-290). Frankfurt a. M.: Suhrkamp.

Walzer, M. (1992). Sphären der Gerechtigkeit. Frankfurt a. M.: Campus. 